\title{
Research on Development of Scientific Campus Football Training System in Middle and Primary Schools of Yanbian Prefecture under New Situation
}

\author{
Longhua $\mathrm{Li}^{1}$ Yijun Wang ${ }^{2, *}$ \\ ${ }^{1,2}$ Yanbian University, Yanji, Jilin, 133002, China \\ *Corresponding author
}

Keywords: Middle and primary schools in Yanbian, Campus football, Scientific training, Research.

\begin{abstract}
With the deepening reform of education in China, the requirements for the education and teaching for students become higher and higher. While teaching students the professional knowledge, it is required to pay attention to the improvement of students' comprehensive quality. Since the campus football culture was proposed, people have been paying more and more attention to it. Taking Yanbian Prefecture as an example, the development of the scientific campus football training system in middle and primary schools in this area is analyzed in this paper.
\end{abstract}

\section{Introduction}

In 2009, China University Football Association (CUFA) was held in Qingdao, which marked the formal starting of Chinese teenager campus football activities. Meanwhile, this activity was initiated by the Ministry of Education and the General Administration of Sport of China together by publishing a document for the first time since the establishment of New China, so it was eye-catching in the sports development of middle and primary schools. 44 cities were determined for the arrangement initially in this activity and would be perfected within 10 years. The proposal of this activity not only popularized the related knowledge and skills of football to the teenagers, but also improved their team spirit and struggle consciousness, thus forming a teenager football training system in combination of sports and education sports.

\section{Significance of campus football in middle and primary schools}

The students of middle and primary schools always have a strong passion for the football and are keen on it very much. However, in the development process in the middle and primary schools, affected by the sports fields, financial support and other factors, the football cannot popularized effectively. Due to the lack of football fields, students cannot put the football into practice. Meanwhile, there is no school-based football talent training system, and the technical level of football is not high, so the educators still continue to work hard in the development process of campus football in the middle and primary schools.

\section{Current development situation of football in middle and primary schools in Yanbian}

In the development process of campus football, schools are an important factor, which are not only the developer of the activity, but also the executor. Their development and implementation of the campus football will influence the development of campus football in the middle and primary schools directly. There are many factors influencing the development of the campus football, and their interaction causes the slow development of the campus football in China. Next, the main difficulties on the current situation of the football curriculum and in the development process of football sports in the schools are analyzed. 


\subsection{Current situation of football curriculum in schools}

After the interview and investigation of a large number of primary and middle schools in Yanbian, we have learned that the implementation of the football curriculum in this area is not ideal. Only a few schools have offered the education and teaching of this curriculum, and most schools don't offer this curriculum. There are some reasons. First of all, schools pay too much attention to the education and teaching of students' professional culture knowledge, but ignore the importance of sports. Secondly, due to the lack of appropriate football fields and sports equipment in the middle and primary schools, this curriculum is unable to start. Thirdly, because of many physical contacts with some dangers in the football sports, schools reduce the football sports in order to better avoid the damages caused by football to the students.

The arrangement and setting of PE curriculum in the middle and primary schools is not reasonable. Most schools have less arrangement of PE curriculum, and often pay too much attention to the education of students' professional knowledge such as Chinese and math, but pay less attention to the PE curriculum, especially football. They usually think that students' professional knowledge is the key to reflect the teaching levels of schools, and the sports activities can not bring a better source of students to the schools. Besides, teachers often occupy students' PE curriculum in the teaching process to increase the teaching of professional knowledge. However, it is the key to cultivate students' comprehensive quality in the education reform of the new era. It is necessary to strengthen the training of students' physical quality while improving their professional knowledge, so the setting of PE curriculum must be more scientific and reasonable. Most students in the middle and primary school are very fond of sports, including the football. However, due to the heavy workload of various professional curriculums, they have to give up sports in order to improve their academic performance.

\subsection{Difficulties in development of football in schools}

At present, there are three main difficulties in the development process of football in the middle and primary school of Yanbian area: the first is the lack of funds for the development of football; the second is the lack of football fields and training facilities; the third is the lack of excellent coaches. A survey of the middle and primary schools in Yanbian shows that the football fields are available only in the schools above the county level with relatively good conditions, and most middle and primary schools do not have plastic track-and-field grounds or turf football fields. A standard football field plays an important role in the development of football activities, but most schools can not meet this condition, so there are some difficulties in the development of football activities. In the development process of the football activities, a lot of funds are requited for the construction and maintenance of the fields, but the financial departments of the government have limited funding capacity for the middle and primary schools, which leads to the insufficient funds and restricts of the football activities. In addition, the allocation of the teachers for the football in the middle and primary schools is often imperfect, and there are not enough professional coaches. In the teaching of football curriculum, teachers' ability will affect students' learning and development directly. If teachers' professional knowledge is not solid enough, their ability of teaching and training will be limited, which will lead to the football curriculum to be difficult to play the role effectively. ${ }^{[2]}$

For the current problems on the development of football in the middle and primary schools, the education departments must find the reasonable solutions with the school leaders in time. First of all, be sure to establish a correct concept of running a school and strengthen the school leaders and teachers' understanding of the football. Secondly, the national education department shall increase the economic support for the football in the middle and primary schools to create a good atmosphere for the development of football activities in the middle and primary schools. Finally, the schools must reduce the learning burden of the students in the middle and primary schools and provide more spare time for the students to take appropriate sports, in order to cultivate students' struggle consciousness and teamwork ability in the football games, thus promoting students' comprehensive development. 


\section{Problems in football training in middle and primary schools of Yanbian}

Although there are some problems in the development of football in the middle and primary schools at present, some schools still attach great importance to the football training. These schools organize students to carry out the football training to improve their football skills and physical fitness effectively. However, in actual training, they still are affected by some factors, such as the training time, training frequency, technical and tactical training, and physical training. ${ }^{[3]}$

\subsection{Unreasonable training time}

A survey of some middle and primary schools in Yanbian Prefecture shows that, the time arrangement of football training in most schools is unreasonable. These schools will often arrange a football training class in $\mathbf{4 5}$ minutes. Due to the short time, the students cannot be trained enough and they will be affected in the actual competitions.

\subsection{Limited training frequency}

The football training needs to be accumulated for a long time, but in the actual football training process in the middle and primary schools, the training frequency is limited is five times at most a week. In addition to the short training time every time, students' football skills have not been cultivated effectively in this training mode. Therefore, the football training teachers in the middle and primary school of Yanbian must consider how to increase the training frequency to improve the students' football ability based on not affecting their normal learning. Moreover, Yanbian Prefecture is located in the northeast of China, where it is very cold in winter, so it is unable to carry out the outdoor football training effectively in winter, and students only receive some simple indoor training. This pattern will last about three months every year. Therefore, in this context, the football training frequency for students has been reduced greatly.

\subsection{Lack of physical training}

In the football training process in the middle and primary schools, we must pay attention to the physical training of students. The requirement for the physical fitness of students in the football activities is extremely high, and students will consume a lot of physical energy in 90 minutes of the game. Therefore, we must strengthen the football physical training in the middle and primary schools. However, the students in the middle and primary schools are in an important growth and development process. The excessive physical training will cause damage their bodies. Therefore, teachers shall control the degree of training for the students in the physical training process, not only to improve the students’ physical fitness, but also not to damage students’ somatic functions.

\subsection{Unsystematic technical and tactical training}

In the football training, the training and improvement of skills is the key of training. However, in most middle and primary schools of Yanbian, there are no more excellent coaches to guide the students in football training, so students' skill level is low. Meanwhile, football itself is a team sport, which requires high teamwork consciousness. In order to achieve cooperation better, players need to master the corresponding football tactics, through football tactics to coordinate with each other effectively, and then improve the football level and play the real role of the combination of skills and tactics on the field.

\section{Establishment of scientific training system}

\subsection{Allocate the training time reasonably}

\subsubsection{Arrange the daily training time reasonably}

In the football training for students, the daily training time must be arranged scientifically and reasonably. First of all, coaches shall make full use of the morning to carry out the appropriate 
training for students and control the training time and training intensity, to avoid the excessive time and intensity that will affect the state of students in the daytime classes. Secondly, coaches shall make the most of the big break in the afternoon and the self-study at night for training, which will not only affect the students' daytime classes, so that the targeted training content for students is arranged fully. In addition, coaches can conduct some training for students at weekends. Because the students have plenty of time at weekends, coaches also can conduct some live operational training, and then summarize and communicate after the game.

\subsubsection{Arrange the training in winter and summer vacations reasonably}

The students in the middle and primary schools have relatively long winter and summer vacations, so coaches shall take advantage of this time reasonably to train students. The football training in Yanbian area during the winter vacation can only be carried out indoors, so the training the winter vacation can be carried out in a small indoor range with the technical cooperation training to enhance the good teamwork between the players. The training during the summer vacation shall be more comprehensive. Control the training between 90 and 120 minutes every time as soon as possible and increase the training frequency to improve the players' technical ability and teamwork ability. The training in winter and summer vocations will make the team have more significant progress. ${ }^{[5]}$

\subsection{Increase physical training intensity}

A scientific training system for students mainly consists of two aspects: the daily physical training and the preparation training before games. The daily physical training mainly includes the training of speed, strength, flexibility and endurance. In the preparation before a game, in order to play the game better, coaches need to help students adjust their physical state to the best. In the actual football game, students need a lot of running to play the football skills fully. Therefore, the physical training in this stage shall focus on the endurance training, and the training of speed, strength and flexibility shall be the auxiliary training.

\subsection{Intensify technical training}

Good technical skills are the foundation in the football games, so students must practice the skills repeatedly in the training process to use the football skills flexibly. In the concrete technical training, coaches shall enhance students' accuracy of passing and catching the football and control of strength. In addition, they must strengthen the training of position skill. In the football games, good position arrangement is the key for players to play their roles. The players in different positions need to grasp different position skills, so coaches must carry out the targeted technical training for students according to the specific positions in the technical training.

\section{Summary}

To sum up, the development of campus football is accelerating and catches people's attention gradually under the new situation. There are some problems in the football training for the students in the middle and primary schools, which restrict their football skills to a certain extent. In order to cultivate students' football skills better, it is necessary to strengthen the training with a more scientific training system, so as to improve students' comprehensive quality of football, and promote the comprehensive development of the students in the middle and primary schools and the progress of China's football level.

\section{Acknowledgement}

This research was financially supported by the social science project of the Education Department of Jilin Province in the 13th Five-Year Plan in 2018, Analysis of Current Situation and Countermeasures for Campus Teenager Football in Yanbian under New Situation (Grant NO. JJKH201809020SK). and also supported by the educational science task of Jilin Province in the 13th Five-Year Plan in 2017, Research on Development of Campus Football and Training Mode of 
Teenager Football Talents in Yanbian under New Situation (Grant NO. GH170025).

\section{References}

[1] Xie Chengguang, Research on Current Situation and Development Countermeasures for Cultivation of Teaching Staff of Campus Football in China under New Situation, Beijing Sport University, 2016.

[2] Li Jixia, Research on Development Strategy for Teenager Campus Football in China, Shanghai University of Sport, 2012.

[3] Feng Aimin, Study on the Sustainable Development of Four Levels of the Campus Football League in China in the New Situation, China School Physical Education (higher education), 2016, 3 (04): 6-10.

[4] Gao Yuan, Opinions on Construction of Football Culture University Campus under New Situation, Contemporary Sports Technology, 2017, 7 (26): 64-65.

[5] Liu Ainuo, Research on Differences between Boys in Grade 3-5 Receiving Amateur Football Training and Those Who don't Receive Football Training in Self-awareness and Self-esteem, Xi'an Physical Education University, 2017.

[6] Wang Qing, Research on Evaluation Index System for Scientific Training Ability of Primary-level Football Coaches, Neijiang Technology, 2012, 33 (05):181+193.

[7] Guo Yongliang, Discussion on Basic Skills and Scientific Tactics Training for Teenager Football Players, Journal of Dandong Teachers College 2002 (S1): 49-51.

[8] Liu Tie, Opinions on Speed and Endurance Training of Football from Perspective of Sports Bioscience, Journal of Chengdu Sport University, 2000 (06): 75-78. 